\title{
Patent Arterial Duct and Vascular Ring in a Premature Infant
}

\author{
Anil Bhattarai, ${ }^{1}$ Vladimiro Vida, ${ }^{2}$ Silvia Ricato, ${ }^{1}$ Sabrina Salvadori,, Giovanni Stellin ${ }^{2}$ \\ 'Department of Pediatrics, University of Padua, Italy, ${ }^{2}$ Department of Pediatric and Congenital Cardiac Surgery, University of \\ Padua, Italy.
}

\section{ABSTRACT}

We report a case of a 750 grams premature female who was scheduled for surgical ligation of a patent arterial duct. Intra-operative findings showed a patent arterial duct in association to a retroesophageal aortic arch creating a complete vascular ring around the trachea, which was successfully divided. A vascular ring should be ruled-out in premature infants prior to ductal ligation at bidimensional echocardiography.

Keywords: congenital heart disease; premature infant; surgery; vascular ring.

\section{INTRODUCTION}

Patency of the arterial duct is a common problem in preterm infants and is often associated with an increased hospital morbidity and mortality. ${ }^{1}$ Medical therapy has been proven to be the initial treatment of choice and indomethacin and ibuprofen are the most widely used drugs. However a Patent Arterial Duct (PDA) must be ligated anytime medical treatment has failed or is contraindicated for a prompt improvement in cardiorespiratory failure and better survival to discharge..$^{1,2}$

The association of a patent arterial duct with a right aortic arch with left descending aorta and a retroesophageal aortic segment is an extremely rare congenital vascular malformation which may cause symptoms of compression from a vascular 'ring'.

We report the case of a premature infant with a patent duct and a vascular ring due to a retro-esophageal aortic arch which was successfully divided.

\section{CASE REPORT}

A 13 day-old premature female, born at 24 weeks of gestational age (750 grams of weight), with the diagnosis of patent arterial duct, was transferred from a peripheral hospital to our Institution for surgical arterial duct ligation, after three unsuccessful cycles of medical treatment with ibuprofen. Her post-natal course was complicated by respiratory distress and hyaline membrane disease. A screening echocardiogram showed a moderate size patent arterial duct. In addition, she was intubated, requiring intermittent mandatory ventilatory support for her increasing respiratory compromise and on intravenous infusion of dopamine $(8$ micrograms/kilogram/minute). Repeat echocardiogram at our Institution confirmed the presence of a moderate size patent arterial duct with a moderately dilated left ventricular cavity.

Correspondence: Dr. Vladimiro Vida, Department of Pediatric and Congenital Cardiac Surgery Unit, University of Padua, Via Giustiniani, 2 - 35100 Padua, Italy. Email: vladimirovida@yahoo.it, Phone: $+39-049-8212410$. 
At the age of 20 days, at bedside in Neonatal Intensive Care Unit (NICU) through a routine limited posteriorlateral thoracotomy, the thoracic aorta was exposed and the aortic isthmus dissected. Retro-esophageal right aortic arches with left descending aorta together with a long patent arterial duct were found, resulting in a complete vascular structure around the trachea and the esophagus (Figure 1A).

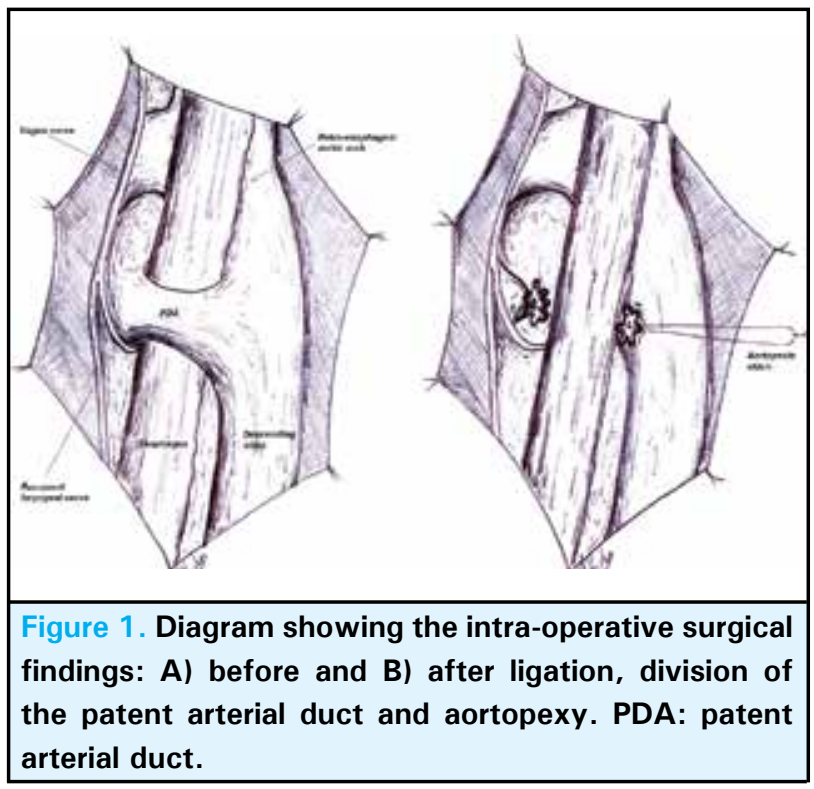

Instead of a routine surgical ligation, the patent arterial duct was temporarily ligated with no hemodynamic or respiratory changes and subsequently it was doubly ligated and divided to interrupt the vascular ring. An aortopexy, by suspending of the aortic stump on the chest wall, to prevent further compression of the mediastinal structures at the time of chest closure, was also performed (Figure 1B). Her postoperative course was uneventful; she was extubated on postoperative day 29 and eventually discharged home two months later.

\section{DISCUSSION}

Right aortic arch with left descending aorta and a retro-esophageal aortic segment is a rare congenital vascular malformation which may cause symptoms of compression from a vascular 'ring'. ${ }^{3,4}$ The presence of this malformation is usually suspected from the clinical signs and barium swallow and then confirmed by angiocardiography or magnetic resonance. ${ }^{3,4}$ However, this diagnosis is extremely difficult to make in very lowweight premature babies where other more frequent causes of respiratory distress can be suspected. ${ }^{1}$

Surgical division of the vascular ring is always necessary in patients presenting with respiratory distress or with esophageal obstruction and an aortopexy can be associated to prevent future compression on the airways and on the esophagus. ${ }^{5-7}$ Our patient had an intra-operative diagnosis of complete vascular ring and nonetheless she did not have a preoperative diagnosis of tracheal compression, a simple ligation of a patent arterial duct was not retained to be the treatment of choice. The baby-girl underwent a vascular ring division and the aortic stump was suspended far away from the trachea to prevent further compression of the trachea possibly jeopardizing airway's structure.

Based on this experience, care should be taken to ruleout the presence of a vascular malformation during routine pre-operative two-dimensional echocardiogram in premature patients with suspect diagnosis of PDA. The proper diagnosis of vascular ring could be missed at the time of the surgical repair and could impair the postoperative course and/or the long-term follow-up. A pediatric cardiologist should be involved in the care of these patients, analyzing the echocardiogram to determine the arch location, the presence of associated lesions such as aortic coarctation or intra-cardiac anomalies that may alter the therapy of the patient.

\section{REFERENCES}

1. Van Overmeire B, Smets K, Lecoutere D, Van de Broek H, Weyler J, Degroote K, et al. A comparison of ibuprofen and indomethacin for closure of patent ductus arteriosus. New Engl J Med. 2000;343:674-81.

2. Vida VL, Lago P, Salvatori S, Boccuzzo G, Padalino MA, Milanesi $\mathrm{O}$, et al. Is there an optimal timing for surgical ligation of patent ductus arteriosus in preterm infants? Ann Thorac Surg. 2009;87:1509-16.

3. Salamat M, Lyon JB. Right aortic arch with anomalous left subclavian artery and left-sided patent ductus arteriosus (vascular ring) in an extremely low-birth-weight infant. Pediatr Cardiol. 2009;30:389-90.

4. Schneeweiss A, Blieden L, Shem-Tov A, Deutsch V, Neufeld HN. Retroesophageal right aortic arch. Pediatr Cardiol. 1984;5:191-6.

5. Gumbiner $\mathrm{CH}$, Howell DM, Kingry RL, Prager RE. Double aortic arch with patent ductus arteriosus in a premature infant: an unusual vascular malformation. Tex Heart Inst J. 1983;10:405-8.

6. Hastings LA, Bushman GA. An unusual cause of arterial desaturation in the premature infant undergoing patent ductus arteriosus ligation. Anesth Analg. 2003;96:692-3.

7. Backer CL, Ilbawi MN, Idriss FS, DeLeon SY. Vascular anomalies causing tracheoesophageal compression: review of experience in children. J Thorac Cardiovasc Surg. 1989;97:725-31. 